\title{
Incorporating Genomic and Genetic Testing into the Treatment of Metastatic Luminal Breast Cancer
}

\author{
Sabine Grill ${ }^{a}$ Evelyn Klein ${ }^{a}$ \\ ${ }^{a}$ Department of Gynecology and Obstetrics, Klinikum Rechts der Isar, Technical University Munich (TUM), \\ Munich, Germany
}

\author{
Keywords \\ Metastatic breast cancer - Genomic testing . \\ Genetic testing · Targeted therapy
}

\begin{abstract}
Background: Treatment of patients with luminal metastatic breast cancer (MBC) has become even more complex over the last few years as molecular profiling has begun to alter disease management. It is well accepted that MBC is not curable but is treatable. Today we are able to prolong progression-free survival and partly overall survival with targeted and more individual treatment strategies adjusted according to the molecular subtype. Summary: Genetic and genomic testing has become therapeutically relevant in luminal $M B C$ and is therefore an integral component within the treatment spectrum. By now, germline testing of $B R C A 1$ and $B R C A 2$ and somatic testing for PIK3CA mutations are inevitable elements in disease management and the current state of the art in luminal MBC patients. Furthermore, testing of ESR1 resistance mutation, ERBB2 mutation, microsatellite instability, and neurotrophic tyrosine receptor kinase (NTRK) gene fusion (mainly in secretory breast cancer) has recently gained increasing attention. However, based on the expanding role of personalized medicine, clinicians are now faced with substantial new challenges and possibly unsuspected possibilities. The following review summarizes current developments in genetic and genomic testing in luminal MBC. Key Messages: In luminal MBC genomics have become an integral component within the spectrum of oncological treatment establishing novel therapeutic facilities. Further developments in treatment personalization adjusted ac-
\end{abstract}

cording to the molecular subtype should become increasingly important in order to enhance the progress of de-escalation of chemotherapy in luminal MBC. However, based on the expanding role of personalized medicine, clinicians are now faced with substantial new challenges and possibly unsuspected possibilities.

๑) 2021 S. Karger AG, Basel

\section{Introduction}

In the area of precision medicine for targeted therapies in metastatic breast cancer (MBC), mutation diagnostics are gaining increasing relevance for treatment decisions. Several gene alterations are currently therapeutically relevant and should be included in molecular panel diagnostics.

\section{Definition of the Terms Genetics and Genomics}

Although these terms are often used interchangeably, there are some substantial differences between genetics and genomics. Genetics is the study of heredity, whereas genomics is defined as the study of genes and their functions, and related techniques $[1,2]$. The primary difference between genomics and genetics is that genetics evaluates the functioning and composition of a single gene [1, $2]$. On the other hand, genomics addresses all genes and their interrelationships in order to identify their combined influence on the growth and development of the organism $[1,2]$. 


\section{Genetic Testing}

\section{Implementation of Genetic Testing in Breast Cancer}

Treatment Strategies

Within the framework of 2 large-scale prospective multicenter studies, a significant benefit of the poly(ADPribose)-polymerase (PARP) inhibitors olaparib and talazoparib over standard therapy (any chemotherapy as doctors' best choice) has recently been shown for patients with a germline (g) BRCA1/2 mutation and HER2-negative $\mathrm{MBC}$ disease $[3,4]$. The high overall response rate (ORR) of $59.9 \%$ with olaparib versus $28.8 \%$ for controls in the standard therapy group in the phase 3 OlympiAD trial led to EMA and FDA approval of this inhibitor for locally advanced or metastatic HER2-negative breast cancer with germline mutations in BRCA1/2 [3]. Equally, talazoparib has been approved by the EMA and the FDA due to the results of the EMBRACA trial, where the response rate was $62.6 \%$ in the talazoparib group versus $27.2 \%$ in the standard-therapy group [4]. The improvement of progression-free survival (PFS) in the above mentioned trials (OlympiAD and EMBRACA) through these 2 PARP inhibitors has led to a paradigm shift within treatment of advanced/metastatic breast cancer.

$B R C A 1$ and $B R C A 2$, as tumor suppressor genes, exhibit a critical role in the maintenance of genomic integrity through homologous recombination, which integrates a complex mechanism of DNA double-strand damage restoration [5]. BRCA1/2 dysfunction results in homologous recombination deficiency and genomic instability, leading to a pathological accumulation of deficient chromosomal rearrangement and atypical cellular replication [6]. According to several preclinical and clinical studies, BRCA-associated cancers are associated with a higher sensitivity to chemotherapy, particularly platinum salts which induce covalent crosslinks in cell DNA and subsequently impair the ability of cells to repair this damage [6]. In consideration of this, it is quite understandable that $B R C A$-associated cancers are more susceptible to these drugs due to their impaired homologous recombination [6]. Further research has focused on the rationale for concomitant inhibition of other DNA repair pathways, particularly the (ADP-ribose) polymerase (PARP) enzyme pathway [5]. PARP1/2 inhibitors, such as olaparib and talazoparib, inhibit the single-strand DNA repair mechanism and therefore act as a personalized targeted treatment in these patients [6].

Considering the potential therapeutic applications, identification of inactivating mutations in the genes $B R C A 1$ and BRCA2 has obtained major importance and contributed to an increased demand for genetic testing.

Currently, the EMA/FDA approval for the PARP inhibitors olaparib and talazoparib is restricted to individuals with $\mathrm{MBC}$ and a germline mutation in BRCA1 or
$B R C A 2$. Therefore, testing should be performed on EDTA blood or salvia. Testing of tumor tissue samples for BRCA $1 / 2$ mutations is still challenging, as a germline mutation cannot be confirmed from these samples and certain types of large genomic rearrangements might be missed [7]. In terms of germline testing, pretest genetic counseling is preferable; however, according to the German genetic diagnostics law, it is sufficient to impart information on germline testing by the treating physician if the referral concerns a therapeutic indication. In a second step, if a germline mutation is confirmed, genetic counseling needs to be offered to the patients and particularly their relatives.

Germline mutations in BRCA1 and BRCA2 occupy a central position in the context of hereditary breast cancer [5]. Female gBRCA 1/2 mutation carriers face a cumulative lifetime risk of $72 \%$ and $69 \%$ for the development of breast cancer, respectively [8]. Identification of a germline mutation leads to clinically actionable implications for both the patient and the family, including predictive genetic testing and subsequently the recommendation for intensified surveillance and potentially prophylactic surgery.

Within the framework of extensive analyses, genotype-phenotype correlations have been found [9]. Breast cancers associated with a gBRCA1 mutation mainly present with a triple negative tumor biology ( 70-80\%) and the majority is classified as basal-like in terms of intrinsic subtypes $[6,9,10]$. gBRCA1-associated cancers evince a higher mitotic index and increased lymphocytic infiltration compared to sporadic cancers [6]. Inter alia, recent studies aim to determine the effect of a combined PARP and checkpoint inhibition [11]. gBRCA2-associated tumors rather resemble sporadic tumors, with the vast majority belonging to the luminal B subtype [10].

When assessing molecular subtyping in an unselected cohort of breast cancer patients, some evidence has been obtained regarding the prevalence rate of $B R C A 1 / 2$ mutations in $\mathrm{MBC}$, which ranges from approximately $7 \%$ for germline mutations to $3 \%$ for somatic (s) mutations in the $B R C A 1 / 2$ genes [12]. Within the subgroup of $\mathrm{g} B R C A 1 / 2$ mutated $\mathrm{MBC}$, a prevalence rate of $12-15 \%$ was given for triple-negative breast cancer; approximately $4 \%$ of the gBRCA1/2 mutations were identified within Her2-enriched tumors and $4-5 \%$ were within the subpopulation of ER-positive, HER2-negative tumors [13-16]. As has already been mentioned, $s B R C A 1$ or $B R C A 2$ mutations were found in around $3 \%$ of $\mathrm{MBC}$. When again stratified by tumor biology, a share of 5\% was detected in triplenegative $\mathrm{MBC}$ and approximately $1-2 \%$ was detected in the subgroup of immunohistochemically ER-positive Her2-negativ cancers $[12,14]$.

As a prospect, at the annual meeting of the American Society of Clinical Oncology (ASCO) 2020, the results of 
the TBCRC 048 study, an investigator-initiated study, were presented. In that study, the response to olaparib in $54 \mathrm{MBC}$ patients with $\mathrm{sBRCA1/2}$ mutations or $\mathrm{g} / \mathrm{s}$ mutations in DNA damage response (DDR) pathway genes other than BRCA1/2 was assessed [17]. Regardless of tumor biology, gPALB2 (partner and localizer of BRCA2) mutations and $s B R C A 1 / 2$ mutations predicted response, respectively, whereas no responses were seen with only a $\mathrm{g} / \mathrm{s}$ ATM or g/s CHEK2 mutation [17]. These results may provide a first indication that PARP inhibition might have some impact on other DDR-genes. However, given the limitation of the small sample size, further evaluation is needed to determine the effectiveness of PARP inhibition on $\mathrm{MBC}$ that carry a somatic $B R C A 1 / 2$ mutation or a non-BRCA g/s DDR gene mutation.

\section{Genomic Testing}

\section{PIK3CA Mutation}

Up to $40 \%$ of patients with luminal breast cancer present with activating mutations in the gene PIK3CA, which induce hyperactivation of the $\alpha$ isoform (p110 $\alpha$ ) of phosphatidylinositol 3-kinase (PI3K) [18]. For patients with hormone receptor (HR)-positive, HER2-negative locally advanced/metastatic breast cancer, endocrine-based therapy regimes, with or without the use of a cyclin-dependent kinase 4 and $6(\mathrm{CDK} 4 / 6)$ inhibitor, represent the current standard of care [18]. However, acquired resistance to endocrine-based therapy is still challenging [18].

Emphasizing the high frequency of PIK3CA mutations and the biological relevance of the signaling pathway, PIK3CA mutations seem to be promising with regard to targeted therapies [7]. However, several clinical studies investigating the effectiveness of broad-spectrum panPIK3CA inhibitors like buparlisib have frequently reported adverse events (AE). Particularly AE like diarrhea, transaminitis, hyperglycemia, mood disorders, and rash limited the treatment response regarding discontinuation of use [7].

Compared to this, the application of selective, isoform-specific inhibitors such as alpelisib has mainly overcome these limitations [7]. Results from the phase $3 \mathrm{SO}$ LAR I trial indicate that male and postmenopausal female luminal breast cancer patients carrying PIK3CA mutations benefit from treatment with alpelisib in combination with fulvestrant [18]. The trial enrolled a total of 572 patients, 341 of whom were confirmed to have a PIK3CA mutation, tested on tumor-tissue. In the PIK3CA mutated cohort, a progression-free survival (PFS) of 11 months at a median follow-up of 20 months was reported in the alpelisib-fulvestrant group versus 5.7 months in the placebo-fulvestrant group [18]. The ORR in the PIK3CA mutated cohort was greater with alpelisib-fulvestrant than with placebo-fulvestrant (26.6 vs. $12.8 \%$ ) [18]. The HR for progression or death was 0.65 in the PIK3CA-mutated cohort compared to 0.85 in the PIK3CA wildtype cohort, indicating that preliminary PIK3CA-mutated cancers benefit from targeted therapy with selective PIK3CA inhibition [18]. However, even with the application of a selective PIK3CA inhibitor, there are several AE that need to be considered. In the overall population, 36.6\% developed hyperglycemia in the alpelisib-fulvestrant group versus $0.7 \%$ in the placebo-fulvestrant group. Additionally there was a considerably higher rate of grade 3 diarrhea (6.7 vs. $0.3 \%)$ and rash (9.9 vs. $0.3 \%$ ) [18]. Therefore, a benefit-risk assessment is required before treatment application.

Furthermore, results from the phase 2 study BYLieve were presented at the ASCO 2020 meeting. The authors commented that the combination of alpelisib and fulvestrant showed a clinically meaningful efficacy even in patients with PIK3CA-mutated, HR-positive, HER2-negative advanced breast cancer who have progressed on prior CDK4/6 inhibitor and aromatase inhibitor (AI) treatment [19]. Apart from the existing FDA approval, alpelisib in combination with fulvestrant has recently been approved be the EMA for postmenopausal women and men with HR-positive, HER2-negative PIK3CA-mutated, locally advanced/metastatic breast cancer following endocrine therapy.

Clonal analyses revealed that PIK3CA mutations remain fairly constant between the primary tumor and metastasis, meaning that testing of the primary tumor is feasible for therapy indication [7].

\section{ESR1 Resistance Mutation}

Genomic evolution in the course of tumor progression has been proven [5, 7]. Mutations of estrogen-sensing receptor 1 (ESR1), which result in ligand-independent constitutive ER activity, have been demonstrated to develop in metastases compared to primary tumors. Upon first relapse, ESR 1 mutations are detected in $1-5 \%$ of patients but in up to $40 \%$ of patients who become resistant to AI [20]. ESR1 mutations have been deemed of paramount importance regarding the question of which is the best endocrine partner to CDK4/6 inhibitors in the first line for ER-positive HER2-negative MBC, as they confer resistance to AI but not to selective estrogen receptor degraders [20].

To answer this question, Fribbens et al. [21] conducted a prospective-retrospective analysis of 2 phase 3 randomized trials (SoFEA and PALOMA 3) and evaluated the impact of ESR1 mutations on sensitivity to standard therapies.

In the SoFEA trial, ESR1 mutated patients (tested in ctDNA) treated with exemestan demonstrated a median PFS of 2.6 months; in contrast, the median PFS was 5.7 
months for those given fulvestrant $(\mathrm{HR}=0.52 ; 95 \% \mathrm{CI}$ $0.30-0.92 ; p=0.02$ ) [21]. Contrastingly, there was no significant difference between exemestan and fulvestrant in the cohort of ESR1 wild-type patients (median PFS: 8.0 vs. 5.4 months), respectively ( $\mathrm{HR}=1.07$; $95 \%$ CI 0.68 $1.67 ; p=0.77$ ) [21]. However, ESR1 mutation was not predictive of the efficacy of palbociclib (interaction test; $p=0.74$ ) in the PALOMA 3 trial [21].

The PADA-1 phase 3 trial evaluated the value of monitoring the onset of ESR1 mutations in cell-free DNA on 1,017 MBC patients receiving treatment with an $\mathrm{AI}$ and the CDK4/6 inhibitor palbociclib in the first line [20]. However, the study's findings confirm a low rate of ESR1 mutations in HR-positive, Her2-negative MBC patients without a prior resistance to AI therapy. Throughout treatment with palbociclib and AI, a fast elimination of ESR1 mutations was shown. Even though ESR1 mutation positivity was associated with a shorter median PFS of 17.5 months under the AI-palbociclib regime at a median follow-up of 12.4 months compared to ESR1 wild-type patients (the median was not reached) with an estimated HR of 2.8, the authors indicated that the AI-palbociclib combination retains a clinical activity in this population considering the median PFS of 17.5months [20]. Concluding, ESR1 mutation positivity at baseline could accelerate the onset of resistance to AI-palbociclib, yet clinical implications remain further research. It should be stressed that in the phase 2 PARSIFAL trial no differences ORR were observed between a combination of palbociclib with letrozole and fulvestrant, respectively, in endocrine-sensitive, ER-positive, HER-2-negative $\mathrm{mBC}$ [22].

Whether testing for ESR1 mutation will be a part of future routine molecular profiling protocols remains to be determined. However, if an ESR1-mutation has incidentally been found, the use of selective estrogen receptor degraders rather than $\mathrm{AI}$ might be preferred.

\section{Neurotrophic Tyrosine Receptor Kinase Gene Fusion}

The tropomyosin receptor kinase (Trk) family encompasses the 3 transmembrane proteins TrkA, TrkB, and TrkC [23]. These are encoded by the neurotrophic tyrosine receptor kinase-1 (NTRK1), NTRK2, and NTRK3 genes, respectively [23]. Chromosomal aberrations involving NTRK genes can result in gene fusions that lead to transcription of chimeric TRK proteins, provoking a constitutively activated or overexpressed kinase function with oncogenic potential $[23,24]$. These genetic abnormalities have recently emerged as targets for cancer therapy, because novel compounds have been developed that are selective inhibitors of the constitutively active rearranged proteins [24]. In a basket trial, the selective panTRK inhibitor, i.e., larotrectinib showed activity against TRK fusion-positive cancers [25].The study enrolled 55 patients, including 12 pediatric patients and 43 adults (thereof $1 \mathrm{BC}$ patient); fusions involved NTRK1 $(n=25)$, $\operatorname{NTRK2}(n=1)$, and NTRK3 $(\mathrm{n}=29)$ and 14 unique partners $[25,26]$. The ORR was $75 \%$. At the 12 -month follow up, $71 \%$ of the responses were ongoing and $55 \%$ of the patients remained progression free [25]. The median duration of response and the median PFS had not been reached [25]. None of the patients discontinued larotrectinib due to drug-related AE [25].

Larotrectinib has been approved by the EMA and the FDA for the treatment of adult and pediatric patients with nonresectable/metastatic solid tumors that display an NTRK gene fusion.

There are multiple methods for identifying NTRK fusions, including pan-TRK immunohistochemistry, fluorescence in situ hybridization, and sequencing methods, each with its respective advantages and drawbacks [27].

The incidence of NTRK fusions depends on the cancer entity. Histology-based triaging should be carried out first to separate the rare cancer subtypes that commonly have NTRK fusions from those that have a low pretest probability of NTRK fusions [27]. A potential diagnostic algorithm for NTRK testing is shown is Figure 1. Breast cancer (except the secretory subtype) has a low probability $(<1 \%)$ of harboring NTRK fusions [27].

Rare cancer subtypes that commonly harbor NTRK fusions include secretory carcinomas of the breast and salivary glands, apart from particularly pediatric tumors (e.g., fibrosarcoma) [27]. Secretory breast carcinoma is a very rare and distinct subtype of breast cancer, defined by the presence of intracellular and extracellular secretory material, for which a good clinical outcome is characteristic [28]. For these tumors, pan-TRK immunohistochemistry can be used as an initial screen but, if negative, additional testing with FISH or RNA level fusion should be used.

\section{ERBB2 and ERBB3 Mutations}

Breast cancer patients with ERBB2 and ERBB3 mutations in the absence of HER2 amplification might be promising candidates for targeted therapy with the irreversible pan-HER tyrosine kinase inhibitor neratinib [7, 29]. ERBB2 is overexpressed in up to $20 \%$ of all breast cancer cases, for which the effectiveness of neratinib has already been proven [30]. There is, however, a small percentage of breast cancers (i.e., 2-4\%) that, while not overexpressing ERBB2, develop tumors dependent on this gene.

The phase 2 SUMMIT basket trial investigated the effectiveness of neratinib on multihistology solid tumors with $s E R B B 2$ and $s E R B B 3$ mutations, respectively [29]. In total, 141 patients ( 125 with HER2-mutant tumors and 16 with HER3-mutant tumors) were enrolled into the trial, and $\mathrm{BC}$ was the second most frequent tumor type $(n=25$, $17.7 \%)$. In the sERBB2-mutant BC cohort, single-agent 


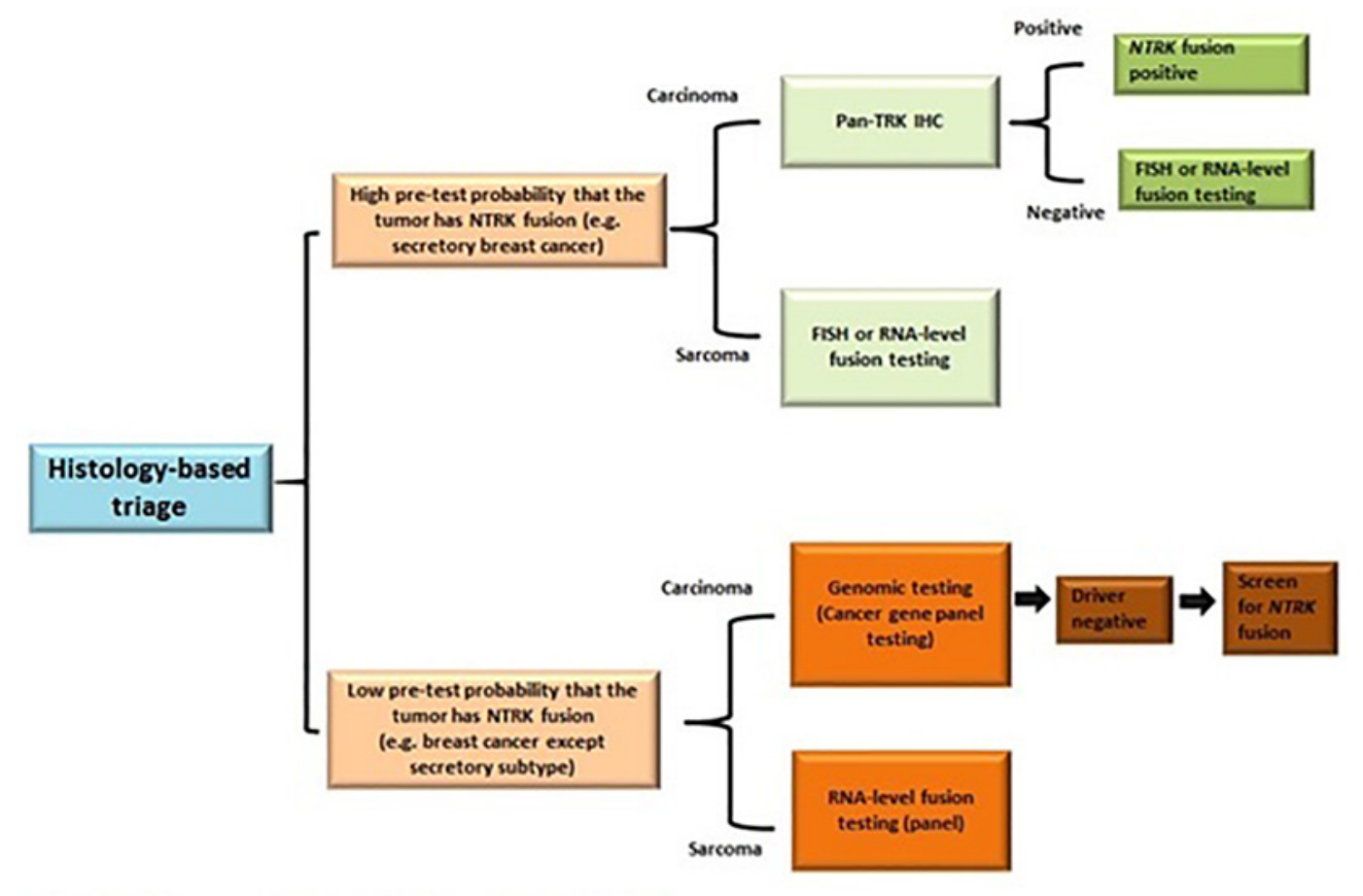

Adapted by Solom on et al, ESMO 201 9,30(Supp!_8)-viiil6-vii22. (27)

Fig. 1. Potential diagnostic algorithm for NTRK testing. For rare cancer subtypes, such as secretory breast cancer, pan-TRK IHC can be used as an initial screening. Extended FISH or RNA-level fusion testing should be used, if IHC is negative. The majority of carcinomas reveal a low probability of NTRK fusion. Molecular testing (e.g., DNA-based cancer gene panels) can be applied to identify driver mutations, which can be used to narrow down the tumors that should be further examined for oncogenic fusions [27]. NTRK fusions are characteristically mutually exclusive, with other common mitogenic driver alterations that activate MAPK signaling [27]. Therefore, carcinomas that are "driver negative" are rather likely enriched for NTRK fusions and these can be screened for by IHC or an RNA-based fusion panel assay [27]. activity for neratinib was observed, with an ORR of $24 \%$, a clinical benefit rate of $40 \%$, and a median PFS of 3.5 months [29]. Another single-arm phase 2 trial confirmed clinical activity in $\mathrm{sERBB2}$-mutated $\mathrm{BC}$ patients, yet there was no response to neratinib in the negative control arm, including the subgroup of $s E R B B 3$-mutated and ERBB2 wild-type BC patients [31].

\section{Microsatellite Instability}

Microsatellite instability (MSI) is a phenotype resulting from a defect in mismatch repair (MMR) genes (MSH2, MSH6, MLH1, and PMS2). MMR-deficient tumors harbor hundreds to thousands of somatic mutations that encode potential neoantigens, known as the MSI-H/hypermutated phenotype and characteristically related to a high tumor mutational burden (TMB-H) [32]. A high neoantigen expression leads to autologous immune recognition of cancer cells by triggering upregulation of immune checkpoint proteins [32].

The MSI phenotype is well recognized in some colon, gastric, pancreatic, and endometrial cancers, yet it is rare in breast cancer and reports are inconsistent [33]. The phase 2 KEYNOTE-158 study examined the anti-programmed death-1 monoclonal antibody pembrolizumab in patients with previously treated, advanced noncolorectal MSI-H/dMMR cancer [32]. As published in 2019, among 233 enrolled patients, 27 different tumor types were included, with endometrial, gastric, cholangiocarcinoma, and pancreatic cancers being the most common noncolorectal cancers [32]. In total, 5 breast cancer patients were included. An objective response rate of 34.3\% (95\% CI 28.3-40.8\%) was reported for the total cohort, with a median PFS of 4.1 months (95\% CI 2.4-4.9 months) and a median overall survival of 23.5 months (95\% CI 13.5 months to not reached) [32]. Considering the breast cancer cases, 2 of the 5 patients included showed a partial response [32].

Pembrolizumab has already been approved by the FDA for the treatment of adult and pediatric patients with unresectable or metastatic MSI-H or dMMR solid tumors. This approval has recently been expanded to unresectable or metastatic solid tumors that are tissue TMB- 


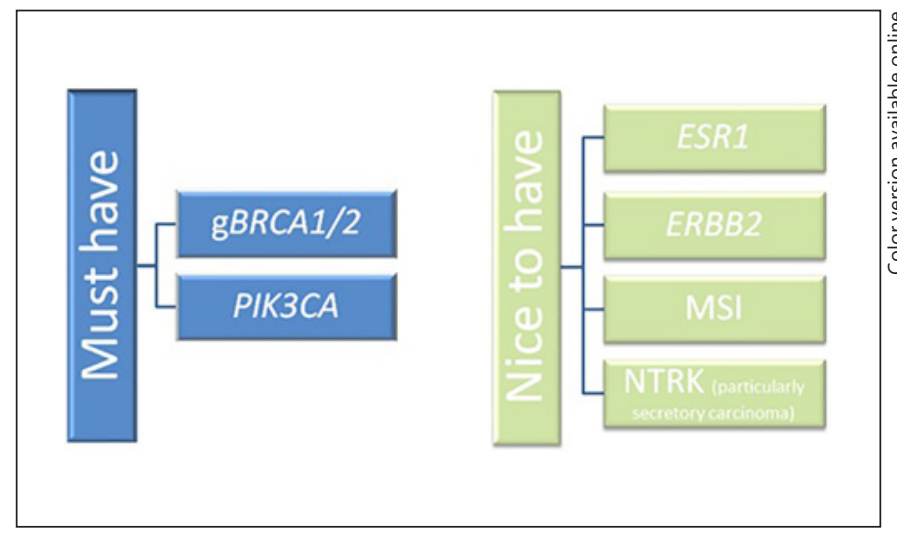

Fig. 2. Recommendation on genetic and genomic testing in standard therapy of luminal MBC. Schematic overview of a testing algorithm for treatment management of luminal MBC.

$\mathrm{H}$ ( $\geq 10$ mutations/megabase) and have progressed following prior therapy and who have no satisfactory alternative treatment options. The approval is based in part on a retrospective evaluation of the phase 2 KEYNOTE-158 trial, in which a link was established between TMB-H status and improved ORR with pembrolizumab in 755 patients with various solid tumors. Of these, 120 (15.9\%) were TMB-H and 15 of those 120 patients (12.5\%) were MSI-H.

In line with this, the phase 3 KEYNOTE-119 study suggested a potential positive association between TMB ( $\geq 10$ mutations/megabase) and clinical benefit with pembrolizumab but not chemotherapy in patients with metastatic triple-negative breast cancer; however, this was limited by the sample [34]. Data remains unknown regarding the benefit of luminal breast cancer.

\section{Tumor Profiling within a Molecular Tumor Board}

Cancer patients for whom gold-standard therapies have been ineffective may be referred for wide-spectrum molecular analysis to potentially identify targets for genomic-based therapies [35]. The introduction of nextgeneration sequencing in clinical practice has opened new opportunities for precision oncology [35]. However, the systematic interpretation of large amounts of next-generation sequencing data and particularly translation of molecular alterations into clinical indications remain challenging $[35,36]$. This makes it essential to standardize the reporting and interpretation of clinically relevant genomics data within the implementation of molecular tumor boards $[35,36]$. These combine the expertise of oncology specialists, (molecular) pathologists, and human genetics and ought to operate as major mediators toward the development of precision oncology [35].
In the pilot study SOLTI-1301 (a nation-wide molecular screening program for patients with $\mathrm{MBC}$ ), the success rate of matching a DNA alteration to an experimental drug or drug class was determined in MBC [37]. Two hundred MBC patients were enrolled, including 192 individuals with luminal breast cancer [37]. Each patient was reviewed by a multidisciplinary advisory board, which recommended potential targeted treatment options [37]. In the total cohort, 116 patients (44.6\%) presented with at least 1 gene mutation (range 1-6) and were matched to targeted therapies. Most frequently mutations in PIK3CA (34\%), TP53 (22\%), AKT1 (5\%), ESR1 (3\%), and ERBB2 (3\%) were identified [37]. In 47 patients $(18.1 \%)$ a mutation was found that was not targetable and $97(37.3 \%)$ individuals did not reveal any mutation [37].

Even though these results are promising, further studies are required to investigate whether a more comprehensive molecular characterization including proteomics may increase therapeutic options for patients with or without somatic mutations [37].

\section{Conclusion}

Genomics have become an integral component in the spectrum of oncological treatment establishing novel therapeutic facilities. Breast cancer in particular is an extremely heterogeneous and complex disease, and molecular profiling has begun to alter disease management on multiple levels (Fig. 2). Further developments in treatment personalization adjusted according to molecular subtype should become increasingly important in order to enhance the progress of de-escalation of chemotherapy in luminal MBC. However, based on the expanding role of personalized medicine, clinicians are now faced with substantial new challenges and possibly unsuspected possibilities.

\section{Conflict of Interest Statement}

The authors have no conflict of interests to declare.

\section{Funding Sources}

There was no funding.

\section{Author Contributions}

Both of the authors wrote this paper. 


\section{References}

1 World Health A. Fifty-seventh World Health Assembly. Geneva, 17-22 May 2004: resolutions and decisions, annexes. Geneva: World Health Organization; 2004

2 World Health Organization. Advisory Committee on Health R. Genomics and world health: report of the Advisory Committee on Health Research. Geneva: World Health Organization; 2002.

3 Robson M, Im SA, Senkus E, Xu B, Domchek SM, Masuda N, et al. Olaparib for Metastatic Breast Cancer in Patients with a Germline BRCA Mutation. N Engl J Med. 2017 Aug; 377(6):523-33.

4 Litton JK, Rugo HS, Ettl J, Hurvitz SA, Gonçalves A, Lee KH, et al. Talazoparib in Patients with Advanced Breast Cancer and a Germline BRCA Mutation. N Engl J Med. 2018 Aug; 379(8):753-63.

5 Hamdan D, Nguyen TT, Leboeuf C, Meles S, Janin A, Bousquet G. Genomics applied to the treatment of breast cancer. Oncotarget. 2019 Jul;10(46):4786-801.

6 Exman P, Barroso-Sousa R, Tolaney SM. Evidence to date: talazoparib in the treatment of breast cancer. OncoTargets Ther. 2019 Jul;12 5177-87.

7 Tendl KA, Bago-Horvath Z. Molecular profiling in breast cancer: ready for clinical routine? Mag Eur Med Oncol. 2020;13(4):445-9.

8 Kuchenbaecker KB, Hopper JL, Barnes DR, Phillips KA, Mooij TM, Roos-Blom MJ, et al.; BRCA1 and BRCA2 Cohort Consortium. Risks of Breast, Ovarian, and Contralateral Breast Cancer for BRCA1 and BRCA2 Mutation Carriers. JAMA. 2017 Jun;317(23):240216.

9 Mavaddat N, Barrowdale D, Andrulis IL Domchek SM, Eccles D, Nevanlinna H, et al.; HEBON; EMBRACE; GEMO Study Collaborators; kConFab Investigators; SWE-BRCA Collaborators; Consortium of Investigators of Modifiers of BRCA1/2. Pathology of breast and ovarian cancers among BRCA1 and BRCA2 mutation carriers: results from the Consortium of Investigators of Modifiers of BRCA1/2 (CIMBA). Cancer Epidemiol Biomarkers Prev. 2012 Jan;21(1):134-47.

10 Wittersheim M, Büttner R, Markiefka B. Genotype/Phenotype correlations in patients with hereditary breast cancer. Breast Care (Basel). 2015 Feb;10(1):22-6.

11 Peyraud F, Italiano A. Combined PARP Inhibition and Immune Checkpoint Therapy in Solid Tumors. Cancers (Basel). 2020 Jun; 12(6):1502.

12 Winter C, Nilsson MP, Olsson E, George AM, Chen Y, Kvist A, et al. Targeted sequencing of BRCA1 and BRCA2 across a large unselected breast cancer cohort suggests that one-third of mutations are somatic. Ann Oncol. 2016 Aug;27(8):1532-8.

13 Cancer Genome Atlas Network. Comprehensive molecular portraits of human breast tumours. Nature. 2012 Oct;490(7418):61-70.

14 Billar JA, Dueck AC, Stucky CC, Gray RJ, Wasif N, Northfelt DW, et al. Triple-negative breast cancers: unique clinical presentations and outcomes. Ann Surg Oncol. 2010 Oct; 17(S3 Suppl 3):384-90.
15 Eccles DM, Li N, Handwerker R, Maishman T, Copson ER, Durcan LT, et al. Genetic testing in a cohort of young patients with HER2amplified breast cancer. Ann Oncol. 2016 Mar;27(3):467-73.

16 Gong Y, Liu YR, Ji P, Hu X, Shao ZM. Impact of molecular subtypes on metastatic breast cancer patients: a SEER population-based study. Sci Rep. 2017 Mar;7(1):45411.

17 Tung NM, Robson ME, Ventz S, Santa-Maria CA, Marcom PK, Nanda R, et al. TBCRC 048: A phase II study of olaparib monotherapy in metastatic breast cancer patients with germline or somatic mutations in DNA damage response (DDR) pathway genes (Olaparib Expanded). J Clin Oncol. 2020;38(15 suppl): 1002.

18 André F, Ciruelos E, Rubovszky G, Campone M, Loibl S, Rugo HS, et al.; SOLAR-1 Study Group. Alpelisib for PIK3CA-Mutated, Hormone Receptor-Positive Advanced Breast Cancer. N Engl J Med. 2019 May;380(20): 1929-40.

19 Rugo HS, Lerebours F, Ciruelos E, Drullinsky P, Borrego MR, Neven P, et al. Alpelisib $($ ALP) + fulvestrant (FUL) in patients (pts) with PIK3CA-mutated (mut) hormone receptor-positive $(\mathrm{HR}+)$, human epidermal growth factor receptor 2-negative (HER2-) advanced breast cancer (ABC) previously treated with cyclin-dependent kinase 4/6 inhibitor (CDKi) + aromatase inhibitor (AI): BYLieve study results. J Clin Oncol. 2020; 38(15 suppl): 1006

20 Bidard FC, Callens C, Dalenc F, Pistilli B, Rouge TD, Clatot F, et al. Prognostic impact of ESR1 mutations in ER+ HER2- MBC patients prior treated with first line $\mathrm{AI}$ and palbociclib: an exploratory analysis of the PADA-1 trial. J Clin Oncol. 2020;38(15 sup$\mathrm{pl}): 1010$

21 Fribbens C, O’Leary B, Kilburn L, Hrebien S, Garcia-Murillas I, Beaney M, et al. Plasma ESR1 Mutations and the Treatment of Estrogen Receptor-Positive Advanced Breast Cancer. J Clin Oncol. 2016 Sep;34(25):2961-8.

22 Llombart-Cussac A, Pérez-García JM, Bellet M, Dalenc F, Gil MJ, Borrego MR, et al. PARSIFAL: A randomized, multicenter, open-label, phase II trial to evaluate palbociclib in combination with fulvestrant or letrozole in endocrine-sensitive patients with estrogen receptor (ER) [+]/HER2[-] metastatic breast cancer. J Clin Oncol. 2020;38(15 suppl): 1007 .

23 Condorelli R, Mosele F, Verret B, Bachelot T, Bedard PL, Cortes J, et al. Genomic alterations in breast cancer: level of evidence for actionability according to ESMO Scale for Clinical Actionability of molecular Targets (ESCAT). Ann Oncol. 2019 Mar;30(3):365-73.

24 Amatu A, Sartore-Bianchi A, Siena S. NTRK gene fusions as novel targets of cancer therapy across multiple tumour types. ESMO Open. 2016 Mar;1(2):e000023.

25 Drilon A, Laetsch TW, Kummar S, DuBois SG, Lassen UN, Demetri GD, et al. Efficacy of Larotrectinib in TRK Fusion-Positive Cancers in Adults and Children. N Engl J Med. 2018 Feb;378(8):731-9.
26 Hyman DM, Laetsch TW, Kummar S, DuBois SG, Farago AF, Pappo AS, et al. The efficacy of larotrectinib (LOXO-101), a selective tropomyosin receptor kinase (TRK) inhibitor, in adult and pediatric TRK fusion cancers [-LBA.]. J Clin Oncol. 2017;35(18 suppl): LBA2501.

27 Solomon JP, Benayed R, Hechtman JF, Ladanyi M. Identifying patients with NTRK fusion cancer. Ann Oncol. 2019;30(suppl 8):viii16-viii22.

28 Lee SG, Jung SP, Lee HY, Kim S, Kim HY, Kim I, et al. Secretory breast carcinoma: A report of three cases and a review of the literature. Oncol Lett. 2014 Aug;8(2):683-6.

29 Hyman DM, Piha-Paul SA, Won H, Rodon J, Saura C, Shapiro GI, et al. HER kinase inhibition in patients with HER2- and HER3-mutant cancers. Nature. 2018 Feb;554(7691): 189-94.

30 Chan A, Delaloge S, Holmes FA, Moy B, Iwata H, Harvey VJ, et al.; ExteNET Study Group. Neratinib after trastuzumab-based adjuvant therapy in patients with HER2-positive breast cancer (ExteNET): a multicentre, randomised, double-blind, placebo-controlled, phase 3 trial. Lancet Oncol. 2016 Mar;17(3): 367-77.

31 Ma CX, Bose R, Gao F, Freedman RA, Telli ML, Kimmick G, et al. Neratinib efficacy and circulating tumor DNA detection of HER2 mutations in HER2 nonamplified metastatic breast cancer. Clin Cancer Res. 2017;23(19): 5687-95.

32 Marabelle A, Le DT, Ascierto PA, Di Giacomo AM, De Jesus-Acosta A, Delord JP, et al. Efficacy of Pembrolizumab in Patients With Noncolorectal High Microsatellite Instability/Mismatch Repair-Deficient Cancer: Results From the Phase II KEYNOTE-158 Study. J Clin Oncol. 2020 Jan; 38(1):1-10.

33 Anbazhagan R, Fujii H, Gabrielson E. Microsatellite instability is uncommon in breast cancer. Clin Cancer Res. 1999 Apr;5(4):83944.

34 Winer EP, Lipatov O, Im SA, Goncalves A, Muñoz-Couselo E, Lee KS, et al. Association of tumor mutational burden (TMB) and clinical outcomes with pembrolizumab (pembro) versus chemotherapy (chemo) in patients with metastatic triple-negative breast cancer (mTNBC) from KEYNOTE-119. J Clin Oncol. 2020;38(15 suppl):1013.

35 Luchini C, Lawlor RT, Milella M, Scarpa A. Molecular Tumor Boards in Clinical Practice. Trends Cancer. 2020 Sep;6(9):738-44.

36 Mateo J, Chakravarty D, Dienstmann R, Jezdic S, Gonzalez-Perez A, Lopez-Bigas N, et al. A framework to rank genomic alterations as targets for cancer precision medicine: the ESMO Scale for Clinical Actionability of molecular Targets (ESCAT). Ann Oncol. 2018 Sep;29(9):1895-902.

37 Pernas S, Villagrasa P, Vivancos A, Scaltriti M, Rodon J, Burgues O, et al. Primary results of the first nationwide molecular screening program in Spain for patients with advanced breast cancer (AGATA SOLTI-1301 study). Ann Oncol. 2018;29:viii90. 